Sustained resonance: a binary system perturbed by gravitational radiation

This article has been downloaded from IOPscience. Please scroll down to see the full text article.

2000 J. Phys. A: Math. Gen. 33513

(http://iopscience.iop.org/0305-4470/33/3/307)

View the table of contents for this issue, or go to the journal homepage for more

Download details:

IP Address: 128.206.162.204

The article was downloaded on $27 / 09 / 2010$ at $15: 11$

Please note that terms and conditions apply. 


\title{
Sustained resonance: a binary system perturbed by gravitational radiation
}

\author{
C Chicone $\dagger$, B Mashhoon $\ddagger$ and D G Retzloff \\ $\uparrow$ Department of Mathematics, University of Missouri, Columbia, MO 65211, USA \\ \$ Department of Physics and Astronomy, University of Missouri, Columbia, MO 65211, USA \\ $\S$ Department of Chemical Engineering, University of Missouri, Columbia, MO 65211, USA
}

Received 26 July 1999

\begin{abstract}
The general phenomena associated with sustained resonance are studied in this paper in connection with relativistic binary pulsars. We represent such a system by two point masses in a Keplerian binary system that evolves via gravitational radiation damping as well as an external tidal perturbation. For further simplification, we assume that the external tidal perturbation is caused by a normally incident circularly polarized monochromatic gravitational wave. In this case, the second-order partially averaged equations are studied and a theorem of C Robinson is employed to prove that for certain values of the physical parameters resonance capture followed by sustained resonance is possible in the averaged system. We conjecture that sustained resonance can occur in the physical system when the perturbing influences nearly balance each other.
\end{abstract}

\section{Introduction}

Imagine a stable Hamiltonian system that is subjected to an external periodic perturbation as well as a frictional force that continuously takes energy out of the system. If the driving force vanishes on the average, then in general the average behaviour of the system is characterized by damped motion. Under favourable conditions, however, the system could get captured into a prolonged resonance if the driving frequency is commensurate with an internal frequency of the system. In this paper, we study the dynamics of this phenomenon in the context of a simple model of certain relativistic binary systems that are of current interest.

Relativistic binary pulsars are generally expected to occur in some astrophysical environment and the gravitational effect of the surroundings on the binary orbit would be tidal in the first order of approximation. The evolution of the binary orbit under the combined influence of gravitational radiation reaction and the external tidal perturbation can lead to resonance under appropriate conditions [1-6]. Once we average over the external periodic tidal perturbation, the binary pulsar is expected to lose energy to gravitational radiation damping so that the relative orbit would spiral inward unless there is resonance capture. This would come about if the external source deposits energy into the orbit over an extended period of time so as to compensate for the continuous loss of energy due to radiation reaction. This situation, which persists until the system leaves the resonance, can occur if the Keplerian period of the osculating ellipse becomes commensurate with the period of the external perturbation. In this way, the binary pulsar can be captured into resonance with its gravitational environment. Then, the standard data reduction schemes are expected to reveal that during resonance capture the semimajor axis of the binary is not monotonically decreasing due to the emission of gravitational radiation but is instead oscillating about a fixed resonance value since the external 
perturbation continuously deposits energy into the orbit during resonance so as to replenish on average the energy lost to gravitational waves. The system leaves the resonance when this delicate energy balance is significantly disrupted. Such resonances are well known in the solar system, where the damping effects have a different origin (see [7] and the references cited therein). One expects that over time the same kind of phenomenon will be observed in a sufficiently large sample of relativistic binary pulsars [8].

It should be mentioned that there are transient chaotic phenomena that are generally associated with resonance. The transient chaos per se may be difficult to detect observationally due to noise and other complications that exist in the data reduction process [9]. On the other hand, there is numerical evidence for a peculiar chaotic effect, namely chaotic transition, that appears to be associated with transient chaos and involves a sudden transformation in the rate of orbital collapse $[3,5]$. That is, the system makes a transition from one relative orbit to another that collapses much more rapidly. Presumably such a chaotic transition-if it indeed occurs in nature-should be detectable by the timing observations of relativistic binary pulsars. Though the unperturbed binary systems in our investigations always involve standard astronomical masses on Keplerian orbits, it is possible to extend the analysis to geodesic orbits around black holes. Such general relativistic systems involve stable Kepler-like orbits as well as unstable orbits. We expect that our analysis of transient chaos could be extended to the stable orbits, while the occurrence of chaos involving the unstable orbits perturbed by incident gravitational radiation has been demonstrated by Bombelli and Calzetta [10]. These results could be significant for the study of chaotic accretion onto black holes and possible chaotic enhancement in the emission of gravitational waves associated with active black holes.

The purpose of the present work is to show that resonance capture can indeed occur on average for a simplified model of the physical situation that has been described here, since our previous work on sustained resonance has been mainly numerical $[2,4,5]$. Our mathematical model is briefly discussed in the next section. The second-order partially averaged system is given in section 3 . The geometry of resonance capture for the second-order partially averaged system is presented in section 4, and it is shown that resonance capture can indeed occur for this partially averaged model under certain conditions. The proof of a resonance capture mechanism for the full model equations remains an important unsolved problem; instead, we provide numerical evidence for sustained resonance in our model in section 5 .

\section{Newton-Jacobi equation with radiation damping}

In a series of papers [1-6], we have studied the long-term evolution of a Keplerian binary system coupled to a gravitational environment. In fact, the influence of the environment has been replaced —-for the sake of simplicity and definiteness — by the tidal perturbation of an external gravitational wave. More precisely, we have considered the simplest gravitational model for a binary system under radiative perturbations. In the lowest (quadrupole) approximation, the external wave exchanges energy and angular momentum with the binary system but not linear momentum. For the purposes of the present investigation, we limit our considerations to a normally incident plane monochromatic external wave that is circularly polarized. The amplitude of this external perturbation is $\epsilon, 0<\epsilon \ll 1$; it indicates the deviation of the spacetime metric from the Minkowski metric as a consequence of the existence of the incident radiation field. In practice, $\epsilon$ could be extremely small; in fact, direct laboratory detection of gravitational waves from astrophysical sources with $\epsilon \approx 10^{-20}$ is the goal of current experimental efforts. The relative motion of the binary is planar due to the transversality of 
the external wave and is given by

$$
\frac{\mathrm{d}^{2} x^{i}}{\mathrm{~d} t^{2}}+\frac{\kappa x^{i}}{\rho^{3}}+\mathcal{R}^{i}=-\epsilon \mathcal{K}_{i j} x^{j}
$$

where $\boldsymbol{x}(t)=\boldsymbol{x}_{1}-\boldsymbol{x}_{2}$ determines the relative orbit, the indices $i, j \in\{1,2\}, \rho=|\boldsymbol{x}|$, and $\kappa=G_{0}\left(M_{1}+M_{2}\right)$. Here the binary system consists of two point masses $M_{1}$ and $M_{2}$ with the centre of mass of the system at rest at the origin of spatial coordinates, i.e. $x_{1}(t)=M_{2} x(t) /\left(M_{1}+M_{2}\right)$ and $x_{2}(t)=-M_{1} x(t) /\left(M_{1}+M_{2}\right)$. The radiation reaction term is given to lowest order by

$\mathcal{R}=\frac{4 G_{0}^{2} M_{1} M_{2}}{5 c^{5} \rho^{3}}\left[\left(12 \rho^{2} \dot{\vartheta}^{2}-18 \dot{\rho}^{2}-\frac{4 \kappa}{\rho}\right) \dot{\boldsymbol{x}}-\frac{\dot{\rho}}{\rho}\left(36 \rho^{2} \dot{\vartheta}^{2}-14 \dot{\rho}^{2}+\frac{4 \kappa}{3 \rho}\right) \boldsymbol{x}\right]$

where $\rho(t)$ and $\vartheta(t)$ describe the relative motion in terms of polar coordinates in the orbital plane and an overdot indicates differentiation with respect to $t$. The influence of the external radiation on the relative motion is represented in equation (1) by a symmetric and traceless tidal matrix $\mathcal{K}$ evaluated at the position of the centre of mass,

$$
\mathcal{K}=\alpha \Omega^{2}\left[\begin{array}{cc}
\cos \Omega t & \pm \sin \Omega t \\
\pm \sin \Omega t & -\cos \Omega t
\end{array}\right]
$$

where $\Omega$ is the frequency of the external wave and $\alpha$, which is of order unity, is its amplitude. The upper (lower) sign indicates right (left) circularly polarized radiation in the transversetraceless gauge. A general comment is in order here regarding the physical realizability of such a perturbation. We can, for instance, imagine another binary system with a relative orbit that is circular with Keplerian frequency $\Omega / 2$. Then, in the rest frame of this binary's centre of mass and neglecting any dissipation the system emits gravitational radiation of frequency $\Omega$ and the waves that propagate perpendicularly to the orbital plane of the circular binary system are circularly polarized; far from the circular binary, these waves are nearly planar and of the form under consideration here.

In the absence of damping, we have shown that if $\epsilon$ is sufficiently small, the planar relative motion is bounded for all time as a consequence of the Kolmogorov-Arnold-Moser (KAM) theorem [1,2]. The physical reason for this confinement is that the external wave does not monotonically deposit energy into the binary orbit; in fact, the general situation is that energy flows back and forth between the wave and the binary so that on average no net transfer of energy takes place. When the orbital damping is taken into account, the general tendency of the binary system is to collapse; however, under certain circumstances the system could get captured into resonance. The necessary condition for resonance is the commensurability of the Keplerian frequency $\omega$ and the wave frequency $\Omega$; that is, relatively prime integers $m$ and $n$ must exist for which $m \omega=n \Omega$. Sustained resonance would actually come about if a delicate balance could be established between the radiative perturbations.

It is useful to express the equations of motion in dimensionless form as in our previous work [3-5]. To this end, we express all spatial intervals in units of $R_{0}>0$ and all temporal intervals in units of $T_{0}>0$, where the scale parameters $R_{0}$ and $T_{0}$ are connected via $\kappa T_{0}^{2} / R_{0}^{3}=1$. Specifically, we assume that at some 'initial' time the lengthscale $R_{0}$ is the semimajor axis of the binary orbit and $2 \pi T_{0}$ is its period. In terms of polar coordinates $(\rho, \vartheta)$, 
the equations of motion then take the form

$\dot{\rho}=\mathcal{P}_{\rho}$

$\dot{\vartheta}=\frac{\mathcal{P}_{\vartheta}}{\rho^{2}}$

$\dot{\mathcal{P}}_{\rho}=-\frac{1}{\rho^{2}}+\frac{\mathcal{P}_{\vartheta}^{2}}{\rho^{3}}+4 \delta \frac{\mathcal{P}_{\rho}}{\rho^{3}}\left(\mathcal{P}_{\rho}^{2}+6 \frac{\mathcal{P}_{\vartheta}^{2}}{\rho^{2}}+\frac{4}{3 \rho}\right)-\epsilon \alpha \Omega^{2} \rho \cos (2 \vartheta \mp \Omega t)$

$\dot{\mathcal{P}}_{\vartheta}=2 \delta \frac{\mathcal{P}_{\vartheta}}{\rho^{3}}\left(9 \mathcal{P}_{\rho}^{2}-6 \frac{\mathcal{P}_{\vartheta}^{2}}{\rho^{2}}+\frac{2}{\rho}\right)+\epsilon \alpha \Omega^{2} \rho^{2} \sin (2 \vartheta \mp \Omega t)$.

Here $\delta$ is the dimensionless parameter that characterizes radiation damping and is given by

$$
\delta=\frac{4 G_{0}^{2} M_{1} M_{2}}{5 c^{5} T_{0} R_{0}} .
$$

For physical systems, $\delta<2^{1 / 2} / 40$ [3]. We find that $\delta \simeq 10^{-15}$ for the Hulse-Taylor relativistic binary pulsar PSR B $1913+16$; this same value for $\delta$ is also approximately valid for the relativistic binary pulsar PSR B1534 + 12 [11].

To study the general characteristics of system (4), it is useful to express this system in terms of Delaunay's elements. These are the natural action-angle variables for the Kepler problem. At each instant of time $t$, the state of relative motion $(\boldsymbol{x}, \dot{\boldsymbol{x}})$, or equivalently $\left(\rho, \vartheta, \mathcal{P}_{\rho}, \mathcal{P}_{\vartheta}\right)$, defines an osculating ellipse that is tangent to the perturbed motion at $t$. The Delaunay variables $(\ell, \hat{g}, L, G)$ are closely related to the orbital elements of the osculating ellipse. Let $a$ and $e$ be the semimajor axis and the eccentricity of the osculating ellipse, respectively; then, $\mathcal{P}_{\vartheta}^{2}=a\left(1-e^{2}\right), \mathcal{P}_{\rho} \mathcal{P}_{\vartheta}=e \sin \hat{v}$, and $\mathcal{P}_{\vartheta}^{2} / \rho=1+e \cos \hat{v}$, where $\hat{v}$ is the true anomaly of the osculating ellipse. Moreover, let $\hat{u}$ be the corresponding eccentric anomaly; then, the Delaunay elements are defined by $[12,13]$

$\ell:=\hat{u}-e \sin \hat{u} \quad \hat{g}:=\vartheta-\hat{v} \quad L:=a^{1 / 2} \quad G:=\mathcal{P}_{\vartheta}=L\left(1-e^{2}\right)^{1 / 2}$.

Only positive square roots are considered throughout this work. Thus we limit our considerations to orbits with positive orbital angular momentum $G$.

The Delaunay equations of motion can be written as

$$
\begin{aligned}
& \dot{\ell}=\frac{1}{L^{3}}+\epsilon \frac{\partial \mathcal{H}_{\mathrm{ext}}}{\partial L}+\epsilon \Delta \mathcal{R}_{\ell} \\
& \dot{\hat{g}}=\epsilon \frac{\partial \mathcal{H}_{\mathrm{ext}}}{\partial G}+\epsilon \Delta \mathcal{R}_{\hat{g}} \\
& \dot{L}=-\epsilon \frac{\partial \mathcal{H}_{\mathrm{ext}}}{\partial \ell}+\epsilon \Delta \mathcal{R}_{L} \\
& \dot{G}=-\epsilon \frac{\partial \mathcal{H}_{\mathrm{ext}}}{\partial \hat{g}}+\epsilon \Delta \mathcal{R}_{G}
\end{aligned}
$$

where $\Delta=\delta / \epsilon, \mathcal{R}_{D}$, for $D \in\{\ell, \hat{g}, L, G\}$, are radiation damping terms $\dagger[3,4]$, and

$$
\begin{aligned}
\mathcal{H}_{\text {ext }}=\frac{1}{2} \alpha a^{2} \Omega^{2} & \left\{\frac{5}{2} e^{2} \cos (2 \hat{g} \mp \Omega t)\right. \\
& \left.+\sum_{\nu=1}^{\infty} \frac{1}{v}\left[K_{+}^{\nu}(e) \cos (2 \hat{g} \mp \Omega t+\nu \ell)+K_{-}^{\nu}(e) \cos (2 \hat{g} \mp \Omega t-\nu \ell)\right]\right\} .
\end{aligned}
$$

$\dagger$ A misprint in the $\ell$-component of the radiation damping terms in equation (18) of [3] and likewise equation (A2) of [4] should be corrected: in the term proportional to $\frac{1}{r^{2}}$ inside the square bracket, the factor $\left(1+70 L^{2} / 3-29 G^{2} / 2\right)$ must be replaced by $\left(70 L^{2} / 3-27 G^{2} / 2\right)$. 
Here

$$
\begin{aligned}
& K_{ \pm}^{v}(e):=\frac{1}{2} v\left(A_{v} \pm B_{v}\right) \\
& A_{v}:=\frac{4}{v^{2} e^{2}}\left[2 v e\left(1-e^{2}\right) J_{v}^{\prime}(v e)-\left(2-e^{2}\right) J_{v}(v e)\right] \\
& B_{v}:=-\frac{8}{v^{2} e^{2}}\left(1-e^{2}\right)^{1 / 2}\left[e J_{v}^{\prime}(v e)-v\left(1-e^{2}\right) J_{v}(v e)\right]
\end{aligned}
$$

where $J_{v}(x)$ is the Bessel function of the first kind of order $v$ and $J_{v}^{\prime}(x)=\mathrm{d} J_{v}(x) / \mathrm{d} x$. Finally, we note that $\Delta$ will be viewed as a fixed constant; that is, we will only consider perturbations in the $(\epsilon, \delta)$ parameter space along lines with equations of the form $\delta=\Delta \epsilon$.

Let us note that in system (7) there is only one 'fast' angle, namely the mean anomaly $\ell$, and its frequency is the Kepler frequency $\left(\omega=L^{-3}\right)$ of the osculating ellipse. Since $\mathcal{H}_{\text {ext }}$ is explicitly time dependent, resonance occurs when $m \omega=n \Omega$, in which case $L$ becomes fixed and equal to $L_{\star}$. The dynamics of the system at the $(m: n)$ resonance is discussed in the next section. If the system is off resonance, however, the system (7) can be averaged with the result that $\mathcal{H}_{\text {ext }}$ averages out to zero and the binary system simply collapses due to gravitational radiation damping.

\section{Partial averaging}

The average value of $\mathcal{H}_{\text {ext }}$ at the $(m: n)$ resonance is in general nonzero only for $n=1$; otherwise, the binary system simply collapses on average just as it does off resonance. Once the system is captured into a primary $(m: 1)$ resonance, the resonance condition is not rigorously maintained. There are in fact deviations that have general characteristics. To study these, we let

$$
\ell=\frac{1}{L_{\star}^{3}} t+\phi \quad L=L_{\star}+\epsilon^{1 / 2} \mathcal{D}
$$

where $\phi$ and $\mathcal{D}$ are new variables associated with resonance and $\epsilon^{1 / 2}$ is the corresponding small parameter. The average energy exchange due to the external perturbation is generally oscillatory

$$
\left\langle\mathcal{H}_{\mathrm{ext}}\right\rangle=T_{c} \cos m \phi+T_{s} \sin m \phi
$$

whereas the damping is unidirectional; in fact, we expect on the average slow oscillatory behaviour about the resonance manifold. This motion can be described in terms of a general damped pendulum-like equation in $\phi$ with torque exerted by the radiation damping.

The equations of motion averaged around the $(m: 1)$ resonance and expressed to second order in $\epsilon^{1 / 2}$ have been given in [4] for the planar Kepler system under radiative perturbations and in [5] for the corresponding three-dimensional case. Restricting the results of [4] to the circularly-polarized incident wave under consideration here (see equations (18), (19) of [4]), 


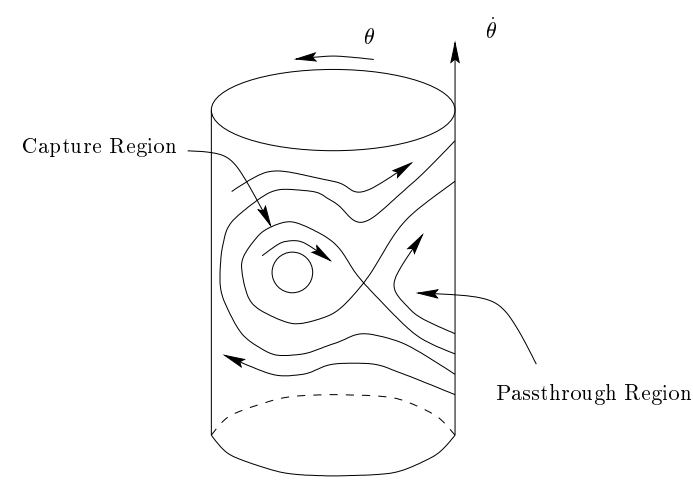

Figure 1. A schematic phase diagram on the phase cylinder for the simple mathematical pendulum with constant torque $\ddot{\theta}-\lambda \sin \theta=\tau$ for the case $\lambda>\tau$.

the second-order partially averaged equations at the $(m: 1)$ resonance are

$$
\begin{aligned}
& \dot{\phi}=-\epsilon^{1 / 2} \frac{3}{L_{\star}^{4}} \mathcal{D}+\epsilon\left(\frac{6}{L_{\star}^{5}} \mathcal{D}^{2}+\frac{\partial T_{c}}{\partial L} \cos m \phi+\frac{\partial T_{s}}{\partial L} \sin m \phi\right) \\
& \dot{\hat{g}}=\epsilon\left(\frac{\partial T_{c}}{\partial G} \cos m \phi+\frac{\partial T_{s}}{\partial G} \sin m \phi\right) \\
& \dot{\mathcal{D}}=-\epsilon^{1 / 2}\left[-m T_{c} \sin m \phi+m T_{s} \cos m \phi+\frac{\Delta}{G^{7}}\left(8+\frac{73}{3} e^{2}+\frac{37}{12} e^{4}\right)\right] \\
& \quad-\epsilon \mathcal{D}\left[-m \frac{\partial T_{c}}{\partial L} \sin m \phi+m \frac{\partial T_{s}}{\partial L} \cos m \phi+\frac{\Delta}{3 L_{\star}^{3} G^{5}}\left(146+37 e^{2}\right)\right] \\
& \dot{G}=-\epsilon\left[\frac{\partial T_{c}}{\partial \hat{g}} \cos m \phi+\frac{\partial T_{s}}{\partial \hat{g}} \sin m \phi+\frac{\Delta}{L_{\star}^{3} G^{4}}\left(8+7 e^{2}\right)\right] .
\end{aligned}
$$

Here $T_{c}=-\hat{f} \cos 2 \hat{g}, T_{s}= \pm \hat{f} \sin 2 \hat{g}$, and

$$
\hat{f}:=\hat{f}(L, G)=-\frac{1}{2} \alpha m L_{\star}^{-6} L^{4} K_{ \pm}^{m}(e)
$$

where $e=\left(1-G^{2} / L^{2}\right)^{1 / 2}$. The general behaviour of the functions $K_{ \pm}^{m}(e)$ has been discussed in [6] (see figure 1 and appendix A of [6]). The functions $K_{-}^{m}(e)$ and $K_{+}^{1}(e)$ are both negative for $0<e<1$. In general, the derivative of the function $e \mapsto K_{-}^{m}(e)$ is negative for $0<e<1$ (see p 107 of [1]). Moreover, for $m>1, K_{+}^{m}(e)$ is positive for $0<e<\hat{e}_{m}$, zero for $e=\hat{e}_{m}$, and negative for $\hat{e}_{m}<e<1$; in fact, $\hat{e}_{2} \simeq 0.76, \hat{e}_{3} \simeq 0.85$, etc.

It is interesting to note that for incident radiation that is circularly polarized, equation (11) implies that $\left\langle\mathcal{H}_{\mathrm{ext}}\right\rangle=-\hat{f} \cos (m \phi \pm 2 \hat{g})$. Introducing a new variable $\theta:=m \phi \pm 2 \hat{g}$ and a new slow temporal parameter $\hat{t}:=-\mu t / b$, where $\mu:=\epsilon^{1 / 2}$ and $b:=L_{\star}^{4} /(3 m)$, system (12) reduces to the form

$$
\begin{aligned}
& \dot{\theta}=\mathcal{D}-\mu\left[\frac{2}{L_{\star}} \mathcal{D}^{2}-b\left(m \hat{f}_{L} \pm 2 \hat{f}_{G}\right) \cos \theta\right] \\
& \dot{\mathcal{D}}=m b \hat{f} \sin \theta+\frac{\Delta b}{G^{7}}\left(8+\frac{73}{3} e^{2}+\frac{37}{12} e^{4}\right)+\mu b \mathcal{D}\left[m \hat{f}_{L} \sin \theta+\frac{\Delta}{3 L_{\star}^{3} G^{5}}\left(146+37 e^{2}\right)\right] \\
& \dot{G}=\mu b\left[ \pm 2 \hat{f} \sin \theta+\frac{\Delta}{L_{\star}^{3} G^{4}}\left(8+7 e^{2}\right)\right]
\end{aligned}
$$

where the overdot now refers to the new time $\hat{t}$ and all relevant quantities are evaluated at $L=L_{\star}$. For example, $\hat{f}_{L}=\left.(\partial \hat{f} / \partial L)\right|_{L=L_{\star}}$, etc. 
It follows from equation (14) that the first-order averaged equations reduce to $G=G_{\star}$, where $G_{\star}$ is a constant, and $\mathcal{D}=\dot{\theta}$, where $\theta(\hat{t})$ is a solution of

$$
\ddot{\theta}-\lambda \sin \theta=\tau \text {. }
$$

Here $\lambda$ and $\tau$ are constants given by

$$
\begin{aligned}
& \lambda=-\frac{1}{6} \alpha m L_{\star}^{2} K_{ \pm}^{m}\left(e_{\star}\right) \\
& \tau=\frac{\Delta b}{G_{\star}^{7}}\left(8+\frac{73}{3} e_{\star}^{2}+\frac{37}{12} e_{\star}^{4}\right)
\end{aligned}
$$

where $e_{\star}=\left(1-G_{\star}^{2} / L_{\star}^{2}\right)^{1 / 2}$. Equation (15) describes a mathematical pendulum with a constant torque $\tau>0$; the phase portrait of this dynamical system is illustrated in figure 1 for $\lambda>\tau$. In this case, there is a homoclinic orbit which encloses a region corresponding to orbits that are captured into resonance. The region outside the homoclinic loop, with the stable and unstable manifolds removed, consists of orbits that pass through the resonance. The presence of the perturbation terms in the system (14) implies that the pendulum is damped (or antidamped) in the second-order averaged equations. That is, averaging the dynamical system to second order results in a pendulum equation of the form (15) with slowly drifting $\lambda$ and $\tau$ as well as an additional term proportional to $\mu \dot{\theta}$. The motion is geometrically described in the next section.

There are several important phenomena associated with resonance: for instance, chaos and resonance capture. Chaotic motion is expected to occur near a resonance under a Hamiltonian perturbation. For non-Hamiltonian perturbations, while chaotic motions are certainly possible, resonance capture is often the dominant phenomenon. At first order, the partially averaged equations at a resonance are generally pendulum-like equations with torque. This nonlinear Hamiltonian system can exhibit chaos and resonance capture under perturbation. The relationship between the chaos in the original dynamical system and possible chaotic effects in the averaged system near $(m: 1)$ resonance is rather subtle and beyond the scope of this work. Here, we will consider instead the possibility of resonance capture. Indeed, we will give a geometric description of resonance capture in system (14) and a rigorous proof that resonance capture does in fact occur.

\section{The geometry of resonance capture and a theorem of Robinson}

We start from our model equations in Delaunay variables (7) and introduce the transformation (10) about a primary resonance; then, the resulting system can be transformed into the system (14) plus terms of higher order by means of an averaging transformation. These higher-order terms may be non-negligible and dealing with this problem is beyond the scope of our work; hence, we consider here resonance capture only in the second-order partially averaged equations and not in the full model. System (14) is typical of the following class of differential equations:

$$
\begin{aligned}
& \dot{\boldsymbol{y}}=F_{0}(\boldsymbol{y}, \boldsymbol{z})+\mu F_{1}(\boldsymbol{y}, \boldsymbol{z})+\mathrm{O}\left(\mu^{2}\right) \\
& \dot{\boldsymbol{z}}=\mu Z_{1}(\boldsymbol{y}, \boldsymbol{z})+\mathrm{O}\left(\mu^{2}\right)
\end{aligned}
$$

where $y \in \mathbb{R}^{2}$ and $z \in \mathbb{R}^{N}$. In particular, note that $z$ is changing on a slow time scale relative to $\boldsymbol{y}$. For system $(14)$, the pair $(\theta, \mathcal{D})$ plays the role of $\boldsymbol{y}$, and $G$, in our case a scalar variable, plays the role of $\boldsymbol{z}$.

For the geometry of resonance capture, let us make a basic hyperbolicity assumption about system (18), namely, let us assume that for each fixed $\zeta$ in some bounded region $\mathcal{Z}$ of $\mathbb{R}^{N}$ the planar differential equation

$$
\dot{\boldsymbol{y}}=F_{0}(\boldsymbol{y}, \zeta)
$$




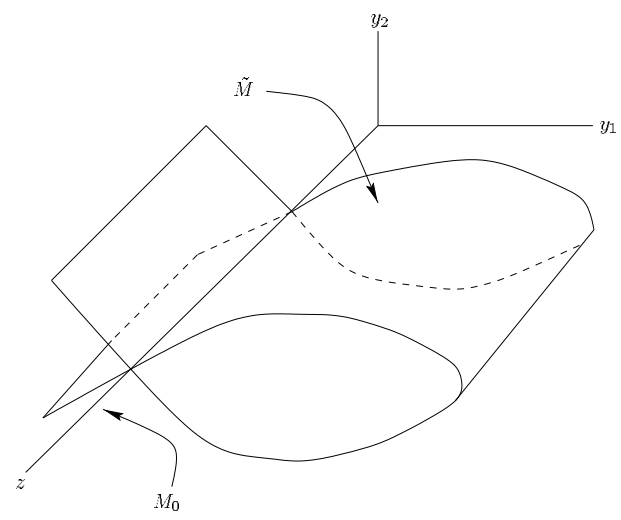

Figure 2. A homoclinic manifold for system (20).

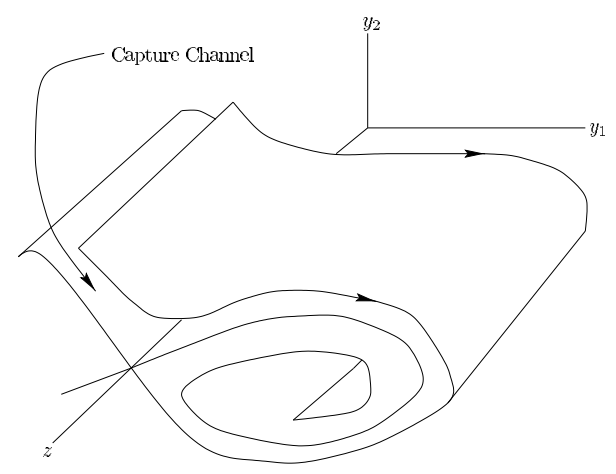

Figure 3. The geometric configuration of the perturbed homoclinic manifold in figure 1 corresponding to resonance capture.

has a hyperbolic saddle point $P(\zeta)$ with a corresponding homoclinic loop given by a solution $t \mapsto \boldsymbol{y}(t, \zeta)$. Then, the set

$$
M_{0}:=\{(\boldsymbol{y}, \boldsymbol{z}): \boldsymbol{y}=P(\boldsymbol{z}), \boldsymbol{z} \in \mathcal{Z}\}
$$

is a normally hyperbolic invariant manifold (consisting entirely of rest points) for the unperturbed system (18), i.e.

$$
\dot{\boldsymbol{y}}=F_{0}(\boldsymbol{y}, \boldsymbol{z}) \quad \dot{z}=0 .
$$

A rough description of this manifold is that nearby orbits are attracted or repelled from the vicinity of $M_{0}$ exponentially fast and the rates of expansion and contraction dominate the corresponding rates for orbits on $M_{0}$. Of course, in our case where the manifold consists of rest points, the second condition is satisfied automatically. The normally hyperbolic manifold $M_{0}$ forms the 'spine' of the submanifold $\tilde{M}$ in $\mathbb{R}^{2} \times \mathbb{R}^{N}$ consisting of the manifold $M_{0}$ - given as a graph over an open subset of $\mathbb{R}^{N}$-and all the corresponding homoclinic loops as illustrated in figure 2. The manifold $\tilde{M}$ 'bounds' a region in the phase space for system (20), and this region corresponds to the initial conditions for orbits that are captured into resonance relative to system (20). When the system is subjected to perturbation, $\tilde{M}$ is expected to 'split', for example, as depicted in figure 3. In fact, by a celebrated theorem of Fenichel [14], the normally hyperbolic manifold $M_{0}$ persists. If the perturbation parameter $\mu$ is sufficiently small, then the perturbed system has an 'invariant' normally hyperbolic manifold $M_{\mu}$ that is again given as a graph over a region in $\mathbb{R}^{N}$; in particular, the manifold $M_{\mu}$ has stable and unstable manifolds. Under appropriate conditions that will be specified below, the stable and unstable manifolds of $M_{\mu}$, the manifolds resulting from the split homoclinic manifold $\tilde{M}$, form a channel in the phase space where orbits of the phase flow enter the vicinity of the resonance (cf figure 3 ). This is the geometric description of resonance capture for a system such as (18).

Let us note that because the unperturbed 'slow manifold' $M_{0}$ consists entirely of rest points, it is an invariant set for the unperturbed flow. However, the perturbed manifold $M_{\mu}$ generally has no fixed points; rather, the flow on $M_{\mu}$ is characterized by a slow drift in the $z$ variable. Thus, $M_{\mu}$ is only invariant in the sense that orbits with initial conditions on this manifold stay on it until they exit at one of its boundary points. Of course, as an effect of the slow drift, an orbit can, after a (perhaps long) sojourn near the resonance, enter a new dynamical regime. The sojourn time can be predicted from an analysis of the flow on the slow 
manifold. Thus, our geometric picture gives a rather complete description of the phenomena associated with resonance capture.

To prove that the geometric description of resonance capture obtains for system (18), we must detect splitting of $\tilde{M}$ and determine the positions of the stable and unstable manifolds of $M_{\mu}$. This is accomplished by an adaptation of Melnikov's method that was first carried out by Robinson [15]. To state his result, let us choose a portion of a plane $\mathcal{L}$ in $\mathbb{R}^{2} \times \mathbb{R}^{N}$ that intersects each homoclinic loop in $\tilde{M}$ perpendicular to the unperturbed vector field on $\tilde{M}$. The choice of $\mathcal{L}$ gives a smooth choice of initial conditions for the unperturbed orbits that correspond to the homoclinic loops. Indeed, for each $\zeta$, consider the corresponding saddle point $P(\zeta)$ and the unperturbed solution $t \mapsto \boldsymbol{y}_{0}(t, \zeta)$ such that $\boldsymbol{y}_{0}(0, \zeta)$ corresponds to the intersection point of $\mathcal{L}$ with the homoclinic loop at $P(\zeta)$. Let $d(\mu, \zeta)$ denote the distance between the intersections of the stable and unstable manifolds of $M_{\mu}$ as measured along $\mathcal{L}$ in the intersecting plane parallel to the $y$ coordinate plane and passing through the point $(P(\zeta), \zeta)$. Also, for two vectors $\boldsymbol{a}, \boldsymbol{b} \in \mathbb{R}^{2}$, let $\boldsymbol{a} \wedge \boldsymbol{b}:=a_{1} b_{2}-a_{2} b_{1}$. The following theorem is proved in [15]:

Theorem 4.1. If system (18) satisfies the basic hyperbolicity assumption and the vector field given by $\boldsymbol{y} \mapsto F_{0}(\boldsymbol{y}, \zeta)$ is divergence-free for each fixed $\zeta \in \mathcal{Z}$, then the splitting distance is given by $d(\mu, \zeta)=\mu d_{1}(\zeta)+\mathrm{O}\left(\mu^{2}\right)$, where the leading-order coefficient is given by the integral

$$
d_{1}(\zeta)=\int_{-\infty}^{\infty}\left(F_{1}+\frac{\partial F_{0}}{\partial z} \frac{\partial z}{\partial \mu}\right) \wedge F_{0} \mathrm{~d} t
$$

all functions in the integrand are evaluated at $\left(\boldsymbol{y}_{0}(t, \boldsymbol{\zeta}), \boldsymbol{\zeta}\right)$, and the function

$$
t \mapsto \frac{\partial \boldsymbol{z}}{\partial \mu}\left(\boldsymbol{y}_{0}(t, \boldsymbol{\zeta}), \boldsymbol{\zeta}\right)
$$

satisfies the variational initial value problem

$$
\dot{Y}=Z_{1}\left(y_{0}(t, \zeta), \zeta\right) \quad Y(0)=0 .
$$

The following immediate corollary is also stated in [15].

Corollary 4.2. If $d_{1}(\zeta)>0$ for all $\zeta \in \mathcal{Z}$, then there is resonance capture. Moreover, the only way a captured solution can leave the vicinity of the resonance is to have its second component, $z$, leave the region where $d_{1}(\zeta)>0$, a process that occurs on a timescale of order $1 / \mu$.

The existence of resonance capture means that a capture channel opens up as in figure 3 and therefore there is sustained resonance.

Let us now formulate and prove a version of another result of Robinson [15] that we use to apply theorem 4.1 to the pendulum-type systems that arise by partial averaging at resonance. Recall that system (14) is derived from the averaged physical model in part by a reversal of time. Thus, it is convenient to have a result that implies 'release from resonance' for system (14) so that it will imply capture into resonance for the original averaged model. Of course, the phrase 'release from resonance' means that the stable and unstable manifolds of the normally hyperbolic manifold $M_{0}$ split in the direction that is opposite to that required for capture; in effect, orbits leave the vicinity of the resonance in a channel bounded by the unstable manifold.

Proposition 4.3. Consider the system

$$
\begin{aligned}
& \dot{\varphi}=v+\mu p(\varphi, v, \boldsymbol{w}) \\
& \dot{v}=f(\boldsymbol{w}) \sin \varphi+g(\boldsymbol{w})+\mu q(\varphi, v, \boldsymbol{w}) \\
& \dot{\boldsymbol{w}}=\mu r(\varphi, v, \boldsymbol{w})
\end{aligned}
$$


where $(\varphi, v, \boldsymbol{w}) \in \mathbb{R} \times \mathbb{R} \times \mathbb{R}^{N}, \mu \geqslant 0$, and all indicated functions are smooth. If there is a point $\boldsymbol{w} \in \mathbb{R}^{N}$ such that

$$
f(\boldsymbol{w})>0 \quad g(\boldsymbol{w})>0 \quad \frac{g(\boldsymbol{w})}{f(\boldsymbol{w})}<1
$$

then the unperturbed system

$$
\dot{\varphi}=v \quad \dot{v}=f(\boldsymbol{w}) \sin \varphi+g(\boldsymbol{w})
$$

has a homoclinic loop that encloses a region $R(w)$ in the $(\varphi, v)$-plane. Suppose that, in addition, $t \mapsto(\varphi(t, \boldsymbol{w}), v(t, \boldsymbol{w}))$ is a solution on the homoclinic loop with

$$
\begin{aligned}
& f^{\prime}(\boldsymbol{w}) r(\varphi(t, \boldsymbol{w}), v(t, \boldsymbol{w}), \boldsymbol{w}) \leqslant 0 \\
& \left(\frac{g}{f}\right)^{\prime}(\boldsymbol{w}) r(\varphi(t, \boldsymbol{w}), v(t, \boldsymbol{w}), \boldsymbol{w}) \geqslant 0
\end{aligned}
$$

where a prime denotes differentiation with respect to $\boldsymbol{w}$, and for $(\varphi, v) \in R(\boldsymbol{w})$,

$$
p_{\varphi}(\varphi, v, \boldsymbol{w})+q_{v}(\varphi, v, \boldsymbol{w}) \geqslant 0 .
$$

If at least one of the inequalities (24), (25), or (26) is strict, then Robinson's integral $I_{R}=d_{1}$ in (21) is negative at $\boldsymbol{w}$. In particular, the perturbed configuration of stable and unstable manifolds corresponds to 'release from resonance'.

Proof. The first statement of the proposition, about the existence of homoclinic loops, is easily proved by elementary phase-plane analysis. Let $s$ denote a new time-like variable given by

$$
\mathrm{d} s=(f(\boldsymbol{w}))^{1 / 2} \mathrm{~d} t
$$

such that $s=0$ at $t=0$ and let $V:=v /(f(\boldsymbol{w}))^{1 / 2}$. In these new variables, system (22) has the form

$$
\begin{aligned}
\frac{\mathrm{d} \varphi}{\mathrm{d} s}=V+\mu & \frac{p\left(\varphi,(f(\boldsymbol{w}))^{1 / 2} V, \boldsymbol{w}\right)}{(f(\boldsymbol{w}))^{1 / 2}} \\
\frac{\mathrm{d} V}{\mathrm{~d} s}=\sin \varphi+\frac{g(\boldsymbol{w})}{f(\boldsymbol{w})} & \quad+\mu\left(\frac{q\left(\varphi,(f(\boldsymbol{w}))^{1 / 2} V, \boldsymbol{w}\right)}{f(\boldsymbol{w})}-\frac{1}{2}(f(\boldsymbol{w}))^{-\frac{3}{2}} f^{\prime}(\boldsymbol{w}) V r\left(\varphi,(f(\boldsymbol{w}))^{1 / 2} V, \boldsymbol{w}\right)\right) \\
& \quad \begin{aligned}
\frac{\mathrm{d} \boldsymbol{w}}{\mathrm{d} s}= & \mu \frac{r\left(\varphi,(f(\boldsymbol{w}))^{1 / 2} V, \boldsymbol{w}\right)}{(f(\boldsymbol{w}))^{1 / 2}} .
\end{aligned}
\end{aligned}
$$

Robinson's integral $I_{\mathrm{R}}$ is then given by

$I_{\mathrm{R}}=\int_{-\infty}^{\infty}\left\{\left(\begin{array}{c}p f^{-1 / 2} \\ q f^{-1}-\frac{1}{2} f^{-3 / 2} f^{\prime} V r\end{array}\right)+\left(\begin{array}{c}0 \\ (g / f)^{\prime}\end{array}\right) \boldsymbol{w}_{\mu}\right\} \wedge\left(\begin{array}{c}V \\ \sin \varphi+g / f\end{array}\right) \mathrm{d} s$

where $\boldsymbol{w}_{\mu}:=\partial \boldsymbol{w} / \partial \mu$. We find that $I_{\mathrm{R}}=I_{1}+I_{2}+I_{3}$, where

$$
\begin{aligned}
& I_{1}:=\int_{-\infty}^{\infty}\left[p f^{-1 / 2}(\sin \varphi+g / f)-V q / f\right] \mathrm{d} s \\
& I_{2}:=\frac{1}{2} \int_{-\infty}^{\infty} f^{-\frac{3}{2}} V^{2} f^{\prime} r \mathrm{~d} s \\
& I_{3}:=-\int_{-\infty}^{\infty} V(g / f)^{\prime} \boldsymbol{w}_{\mu} \mathrm{d} s .
\end{aligned}
$$


Also, let us note that $\boldsymbol{w}$ is constant on the separatrix loop and let us take the solution of system (22) such that the partial derivative $\boldsymbol{w}_{\mu}$ vanishes at $s=0$ as required in the statement of theorem 4.1. The first integral $I_{1}$ can be rewritten as follows:

$$
\begin{aligned}
I_{1} & =\int_{-\infty}^{\infty}\left[p f^{-1 / 2}(\sin \varphi+g / f)-v f^{-1 / 2} q / f\right] f^{1 / 2} \mathrm{~d} t \\
& =\frac{1}{f} \int_{-\infty}^{\infty}[p(f \sin \varphi+g)-v q] \mathrm{d} t .
\end{aligned}
$$

Under the hypothesis (23), the homoclinic loop $\Gamma$ in the $(\varphi, v)$-plane is parametrized with negative orientation relative to the usual orientation of the plane. Also, let us denote by $R$ the bounded region with boundary $\Gamma$ in the $(\varphi, v)$-plane. By Green's theorem and the inequality (26), we have that

$I_{1}=\frac{1}{f} \int_{-\infty}^{\infty}(p \dot{v}-q \dot{\varphi}) \mathrm{d} t=-\frac{1}{f} \int_{\Gamma} p \mathrm{~d} v-q \mathrm{~d} \varphi=-\frac{1}{f} \int_{R}\left(p_{\varphi}+q_{v}\right) \mathrm{d} \varphi \mathrm{d} v \leqslant 0$.

It follows from hypothesis (24) that $I_{2} \leqslant 0$. For the integral $I_{3}$, let us observe that if we take the parametrization of $\Gamma$ such that $V(0)=0$, then $s V(s) \geqslant 0$; that is, $V(s)$ has the same sign as the variable $s$ in the integration. Also, because $\boldsymbol{w}$ is constant for the integration, $\mu=0$ for the functions in the integrand, and hypothesis (25) holds, we have the inequality

$$
\frac{\mathrm{d}}{\mathrm{d} s}\left[\left(\frac{g}{f}\right)^{\prime} \boldsymbol{w}_{\mu}\right]=\left.\left(\frac{g}{f}\right)^{\prime} \frac{\mathrm{d} \boldsymbol{w}_{\mu}}{\mathrm{d} s}\right|_{\mu=0}=\left(\frac{g}{f}\right)^{\prime} r f^{-1 / 2} \geqslant 0 .
$$

Thus, using the fact that $\boldsymbol{w}_{\mu}$ vanishes at $s=0$, note that $(g / f)^{\prime} \boldsymbol{w}_{\mu}$ has the same sign as the variable $s$ for the integration. We conclude that

$$
V\left(\frac{g}{f}\right)^{\prime} \boldsymbol{w}_{\mu} \geqslant 0
$$

over the range of the integration. Thus, $I_{3} \leqslant 0$.

Finally, note that if inequality (26) is strict, then $I_{1}<0$. Likewise, if inequality (24) (respectively inequality (25)) is strict, then $I_{2}<0$ (respectively $I_{3}<0$ ). Hence, $I_{\mathrm{R}}=$ $I_{1}+I_{2}+I_{3} \leqslant 0$ and if at least one of the inequalities (24), (25), or (26) is strict, then $I_{\mathrm{R}}<0$.

To apply proposition 4.3 , let us consider the second-order averaged equations for the normally incident left circularly polarized gravitational wave expressed in decoupled form; namely, system (14) with the 'lower' signs. Also, let us note that this system is a special case of the abstract system in proposition 4.3 once we set $\theta=\varphi, \mathcal{D}=v$, and $G=\boldsymbol{w}$. Recall that if $L_{\star}>G>0$, then $0<e<1$ and $K_{-}^{m}(e)<0$. Hence, we have the desired inequality $\hat{f}\left(L_{\star}, G\right)>0$ once we choose $\alpha>0$. In fact, we set $\alpha=1$. We claim that the hypotheses of proposition 4.3 are satisfied under the following two conditions:

(1) There is a subinterval $\mathcal{G}_{1}$ of the interval $\left(0, L_{\star}\right)$ such that

$$
\frac{\Delta}{m \hat{f} G^{7}}\left(8+\frac{73}{3} e^{2}+\frac{37}{12} e^{4}\right)<1
$$

whenever $G \in \mathcal{G}_{1}$.

(2) There is an interval $\mathcal{G}_{2} \subseteq \mathcal{G}_{1}$ such that the derivative of the function

$$
e \mapsto \frac{1}{\left(1-e^{2}\right)^{7 / 2} \hat{f}}\left(8+\frac{73}{3} e^{2}+\frac{37}{12} e^{4}\right)
$$

is negative on the image of the interval $\mathcal{G}_{2}$ under the transformation

$$
e=\left(1-G^{2} / L_{\star}^{2}\right)^{1 / 2} \text {. }
$$


Condition (1) ensures that hypothesis (23) is satisfied for each $G \in \mathcal{G}_{1}$. Recall that

$$
\hat{f}_{G}:=\frac{\partial \hat{f}}{\partial e} \frac{\partial e}{\partial G}=-\frac{1}{G e}\left(1-e^{2}\right) \frac{\partial \hat{f}}{\partial e}
$$

and since $\partial K_{-}^{m}(e) / \partial e<0$ for $0<e<1$ we have that $\partial \hat{f} / \partial e>0$. Thus, $\hat{f}_{G}<0$ for $G \in \mathcal{G}_{1}$. Turning to the function $r$ in the statement of proposition 4.3, note that

$$
r:=-2 b \hat{f} \sin \theta+\frac{\Delta b}{L_{\star}^{3} G^{4}}\left(8+7 e^{2}\right) .
$$

By the first implication of proposition 4.3, it follows that if $G \in \mathcal{G}_{1}$, then the system

$$
\dot{\theta}=\mathcal{D} \quad \dot{\mathcal{D}}=m b \hat{f} \sin \theta+\Delta \frac{b}{G^{7}}\left(8+\frac{73}{3} e^{2}+\frac{37}{12} e^{4}\right)
$$

has a homoclinic orbit where the corresponding saddle point has coordinates $(\theta, \mathcal{D})=\left(\theta_{\star}, 0\right)$ for some $\theta_{\star}$ in the interval $(3 \pi / 2,2 \pi)$ as long as $\theta \in[0,2 \pi)$. At the boundary of the interval $\mathcal{G}_{1}$, where

$$
\frac{\Delta}{m \hat{f} G^{7}}\left(8+\frac{73}{3} e^{2}+\frac{37}{12} e^{4}\right)=1
$$

the corresponding saddle point with coordinates $\left(\theta_{\star}, 0\right)$ and centre with coordinates $\left(3 \pi-\theta_{\star}, 0\right)$ coalesce at $(3 \pi / 2,0)$; that is, these rest points disappear in a saddle node bifurcation. Thus, there is a subinterval $\mathcal{G}_{2} \subseteq \mathcal{G}_{1}$ such that if $G \in \mathcal{G}_{2}$, then the region $R(G)$, enclosed by the homoclinic orbit, contains only points whose first coordinates $\theta$ are in the interval $\pi<\theta<2 \pi$. In particular, if $G \in \mathcal{G}_{2}$, then $\sin \theta<0$ and therefore $r>0$. Hence, $r \hat{f}_{G}<0$ and hypothesis (24) is satisfied.

To verify hypothesis (25), we must show that if $G \in \mathcal{G}_{2}$, then the derivative of the function

$$
G \mapsto \frac{\Delta}{m \hat{f} G^{7}}\left(8+\frac{73}{3} e^{2}+\frac{37}{12} e^{4}\right)
$$

is positive; however, this requirement follows immediately from condition 2 . For hypothesis (26), let us determine the sign of the divergence of the vector field $\mathcal{V}$ given by

$$
(\theta, \mathcal{D}) \mapsto\left[-\left(\frac{2 \mathcal{D}^{2}}{L_{\star}}+b\left(2 \hat{f}_{G}-m \hat{f}_{L}\right) \cos \theta\right), b \mathcal{D}\left(m \hat{f}_{L} \sin \theta+\frac{\Delta}{3 L_{\star}^{3} G^{5}}\left(146+37 e^{2}\right)\right)\right] .
$$

Indeed, using the inequalities $\sin \theta<0$ and $\hat{f}_{G}<0$, it follows that if $G \in \mathcal{G}_{2}$, then the divergence of $\mathcal{V}$ is the positive quantity

$$
b\left(2 \hat{f}_{G} \sin \theta+\frac{\Delta}{3 L_{\star}^{3} G^{5}}\left(146+37 e^{2}\right)\right) .
$$

This completes the proof of our claim.

To show the desired phenomenon of release from resonance, we will verify conditions 1 and 2. In order to determine the interval $\mathcal{G}_{2}$, let us note that our (unperturbed) pendulum-type planar system has the form

$$
\dot{\theta}=\mathcal{D} \quad \dot{\mathcal{D}}=\lambda \sin \theta+\tau
$$

where $\lambda>0, \tau>0$, and $\tau / \lambda<1$. System (27) is Hamiltonian with total energy

$$
H=\frac{1}{2} \mathcal{D}^{2}+\lambda \cos \theta-\tau \theta \text {. }
$$

Denote the coordinates of its hyperbolic saddle point by $\left(\theta_{\star}, 0\right)$, and recall that $3 \pi / 2<\theta_{\star}<$ $2 \pi$. Also, note that the homoclinic loop is given by the graphs of the functions

$$
\mathcal{D}= \pm 2^{1 / 2}\left(\lambda \cos \theta_{\star}-\tau \theta_{\star}-(\lambda \cos \theta-\tau \theta)\right)^{1 / 2} .
$$


Proposition 4.4. For system (27), there is a point $\hat{\theta}$ such that $\theta_{\star}-2 \pi<\hat{\theta}<\theta_{\star}$ and

$$
\lambda \cos \hat{\theta}-\tau \hat{\theta}=\lambda \cos \theta_{\star}-\tau \theta_{\star} .
$$

The point $(\hat{\theta}, 0)$ is not a rest point. Moreover, the equation

$$
\lambda \cos \theta-\tau \theta=\lambda \cos \theta_{\star}-\tau \theta_{\star}
$$

has no solution for $\theta>\theta_{\star}$. That is, the homoclinic loop at $\left(\theta_{\star}, 0\right)$ crosses the $\theta$-axis at $\theta=\hat{\theta}$ and encloses that portion of the $\theta$-axis given by $\hat{\theta} \leqslant \theta \leqslant \theta_{\star}$. Also, $\hat{\theta}>\pi$ if and only if $k:=\tau / \lambda$ is such that

$$
\frac{1+\left(1-k^{2}\right)^{1 / 2}}{k}+\sin ^{-1} k<\pi
$$

Proof. Define the function $h$ by

$$
h(\theta)=\lambda \cos \theta-\tau \theta-\left(\lambda \cos \theta_{\star}-\tau \theta_{\star}\right)
$$

and note that it has relative maxima at $\theta=\theta_{\star}$ and $\theta=\theta_{\star}-2 \pi$. Also, $h\left(\theta_{\star}\right)=0$ and $h\left(\theta_{\star}-2 \pi\right)=2 \pi \tau>0$. It follows that there is a point $\hat{\theta}$ in the interval indicated in the statement of the proposition such that $h(\hat{\theta})=0$. Moreover, this point is not a rest point. Indeed, the only rest point in the open interval $\left(\theta_{\star}-2 \pi, \theta_{\star}\right)$ is the point $3 \pi / 2-\theta_{\star}$, and it corresponds to a negative relative minimum of $h$. If $\theta>\theta_{\star}$, and $h(\theta)=0$, then there must be some relative maximum of $h$ that is non-negative. However, all the relative maxima that exceed $\theta_{\star}$ have the form $\theta_{\star}+2 \pi N$, where $N$ is a positive integer, and $h$ takes negative values at all such points; therefore, we reach a contradiction. Clearly, $\hat{\theta}>\pi$ if and only if $h(\pi)>0$ or

$$
\tau\left(\theta_{\star}-\pi\right)-\lambda\left(1+\cos \theta_{\star}\right)>0
$$

Recall that $\sin \theta_{\star}=-\tau / \lambda$ and $\cos \theta_{\star}>0$. Thus, with $k:=\tau / \lambda$, inequality (28) is equivalent to the inequality

$$
\frac{1+\left(1-k^{2}\right)^{1 / 2}}{k}+\sin ^{-1} k<\pi
$$

as required.

In summary, let us recall that $f(e)=m b \hat{f}\left(L_{\star}, G\right)$, where $e=\left(1-G^{2} / L_{\star}^{2}\right)^{1 / 2}$ and $\hat{f}\left(L_{\star}, G\right)=-\left(m / 2 L_{\star}^{2}\right) K_{ \pm}^{m}(e)$ by equation (13). For incident left circularly polarized radiation, we have that $f(e)>0$ and $f^{\prime}(e)>0$; in fact,

$$
K_{-}^{m}(e)=-\frac{(5 m+2) m^{m-1}}{2^{m+1}(m+2) !} e^{m+2}+\mathrm{O}\left(e^{m+4}\right)
$$

and for $0<e \leqslant 0.5$ the first term in this Taylor expansion is accurate to about $10 \%$. Then, all the hypotheses of proposition 4.3 are satisfied provided that there is some $e$ such that $0<e<1$ and

$$
\begin{aligned}
& \text { (i) } k(e)=\frac{\Delta}{m L_{\star}^{7}} \frac{1}{f(e)\left(1-e^{2}\right)^{7 / 2}}\left(8+\frac{73}{3} e^{2}+\frac{37}{12} e^{4}\right)<1 \\
& \text { (ii) } k^{\prime}(e) \leqslant 0 \\
& \text { (iii) } \frac{1+\left(1-k^{2}(e)\right)^{1 / 2}}{k(e)}+\sin ^{-1}(k(e))<\pi .
\end{aligned}
$$

One can show by inspection that condition (iii) is satisfied provided that $k_{0}<k(e) \leqslant 1$, where $k_{0} \approx 0.725$. We therefore choose $k(e)=0.9$ in agreement with condition (i). Moreover, one 
can directly verify that $k^{\prime}(e)<0$ if we assume $e=0.5$ and $m=2$. It is interesting to note that the eccentricities of the relativistic binary pulsars PSR B1913 + 16 and PSR B1534 + 12 are $\approx 0.6$ and $\approx 0.3$, respectively. We choose $L_{\star}=\left(\frac{4}{3}\right)^{1 / 2}$, so that $\Omega \approx 1.3$ at the $(2: 1)$ resonance that we wish to consider. Finally, $\Delta$ is uniquely determined from $k(e)=0.9$ once we recall that $\alpha=1$ and $K_{-}^{2}(0.5) \approx-0.009$; hence, $\Delta \approx 5 \times 10^{-4}$.

\section{Discussion}

We have shown that a set of parameters exists such that for incident left circularly polarized radiation the averaged equations permit sustained resonance. The essential requirement for sustained resonance in our approach is that the Robinson integral be nonzero. Our objective has been to prove sustained resonance in the second-order averaged equations for our model. However, we expect that Robinson's integral is generally nonzero without the restrictive assumptions of proposition 4.3. Indeed, it should be clear from the proof of proposition 4.3 that $I_{\mathrm{R}}$ can be negative without the requirement that each of the three integrals $I_{1}, I_{2}$, and $I_{3}$ be negative. For this reason, we expect that resonance capture would generally be possible for either circular polarization state of the incident radiation. Moreover, circular polarization is not essential for sustained resonance as demonstrated by our previous numerical work on resonance capture that did not involve circular polarization $[2,4,5]$. Indeed, the mathematical theory of sustained resonance developed in this paper owes much to the simplification-i.e. the reduction from four to three phase-space dimensions in the system (14) - brought about by the assumption that the normally incident radiation has definite helicity.

The proof of the existence of sustained resonance involves the second-order partially averaged system. We conjecture that the result can be extended to the original system. To provide evidence for this conjecture, it is interesting to use the set of parameters obtained in the previous section as part of the initial conditions needed to integrate the original system numerically. To this end, we integrate system (4) with initial conditions $\left(\rho, \vartheta, \mathcal{P}_{\rho}, \mathcal{P}_{\vartheta}\right)=$ $(1,0,0.5,1), \Omega=2\left(\frac{3}{4}\right)^{3 / 2}, \epsilon=10^{-3}$, and $\delta=\epsilon \Delta=5 \times 10^{-7}$. Thus the initial orbit has $L=\left(\frac{4}{3}\right)^{1 / 2}, e=0.5, G=1$, and $\theta=5 \pi / 3-3^{1 / 2} / 2$, so that $\pi<\theta<2 \pi$ as required. The periastron for this initial osculating orbit, which has true anomaly $\hat{v}=\pi / 2$, occurs at $\vartheta=-\pi / 2$; hence $\hat{g}=-\pi / 2$. In fact, this initial orbit has been used frequently in our previous work [3-6]. We find that with these initial conditions the system is indeed in sustained $(2: 1)$ resonance for either circular polarization state as demonstrated in figures 4 and 5 . Figure 4 can be produced as follows: system (4) is integrated backward from the initial point for normally incident radiation that is left circularly polarized until the system leaves the resonance and a final point of integration is stored; then, the graph is made by integrating forward in time from this point. It is interesting to note that the binary orbit collapses rather rapidly following exit from resonance when the orbit is highly eccentric. This is explained by the fact that the rate of emission of gravitational radiation by the binary orbit is proportional to $\left(1-e^{2}\right)^{-7 / 2}$; hence, as $e \rightarrow 1$ the loss of orbital energy to radiation leads to orbital collapse [3]. Figure 5 can be produced in a similar way and illustrates the same situation for right circular polarization. The possibility of resonance in this case can be seen from the first-order averaged equations (15)(17); that is, $K_{+}^{2}(0.5) \approx 0.923$ and hence $|\lambda|>\tau$, so that the situation depicted in figure 1 holds except that the direction of some arrows should be reversed. It is clear from figures 4 and 5 that evolutionary dynamics of the orbit while trapped in resonance depends sensitively upon the helicity of the incident radiation. For example, the orbit loses angular momentum while in resonance and becomes highly eccentric when the incident radiation has negative helicity as in figure 4 , while the opposite situation holds in figure 5 for positive helicity incident radiation; 

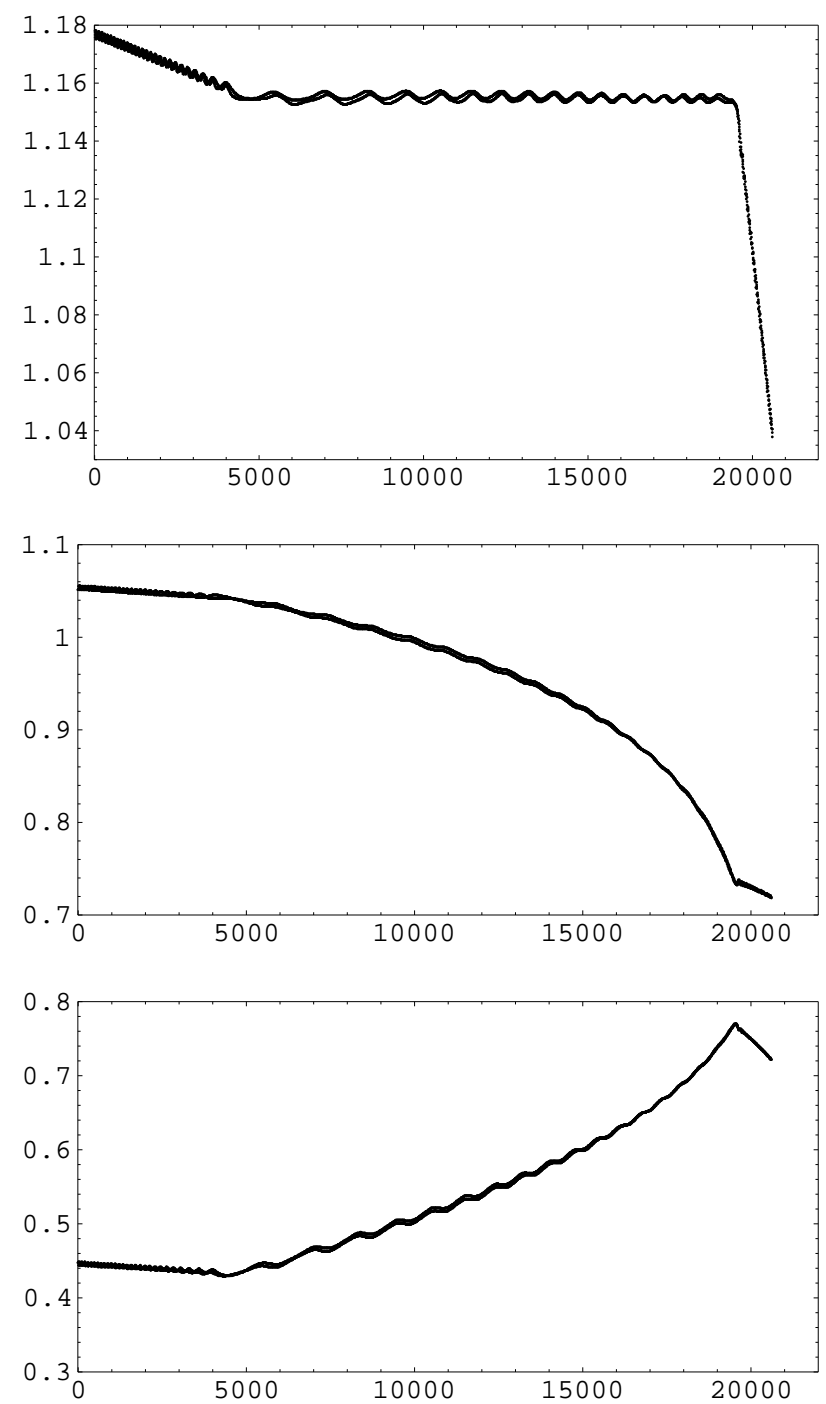

Figure 4. Sustained resonance for the relative orbit in a binary system with initial conditions $\left(\rho, \vartheta, \mathcal{P}_{\rho}, \mathcal{P}_{\vartheta}\right)=(1,0,0.5,1)$ in $(2: 1)$ resonance with a normally incident left circularly polarized gravitational wave. In the graphs, produced by backward and forward integration as explained in the text, this initial point corresponds to $t \approx 9500$. Here $\alpha=1, \epsilon=10^{-3}, \delta=5 \times 10^{-7}$, and $\Omega=2\left(\frac{3}{4}\right)^{3 / 2} \approx 1.299$. The top, middle, and bottom panels depict, respectively, $L, G$, and $e$ versus time. The rate of orbital decay after the resonance is generally different from that before the resonance, since during resonance capture the orbit exchanges angular momentum with the incident radiation such that the orbit has a different eccentricity when it exits the resonance. The duration of the resonance is $\approx 15 / \epsilon$ time units, in agreement with corollary 4.2.

this circumstance may be naturally interpreted in terms of the (negative or positive) angular momentum carried by the (negative or positive helicity) radiation and deposited into the binary orbit. The fact that the amplitude of oscillations around resonance is generally larger when the sense of the helicity of the radiation is the same as the rotation of the orbit is in agreement with our previous work on the Hill system. In contrast to the Hill system, the present case involves 

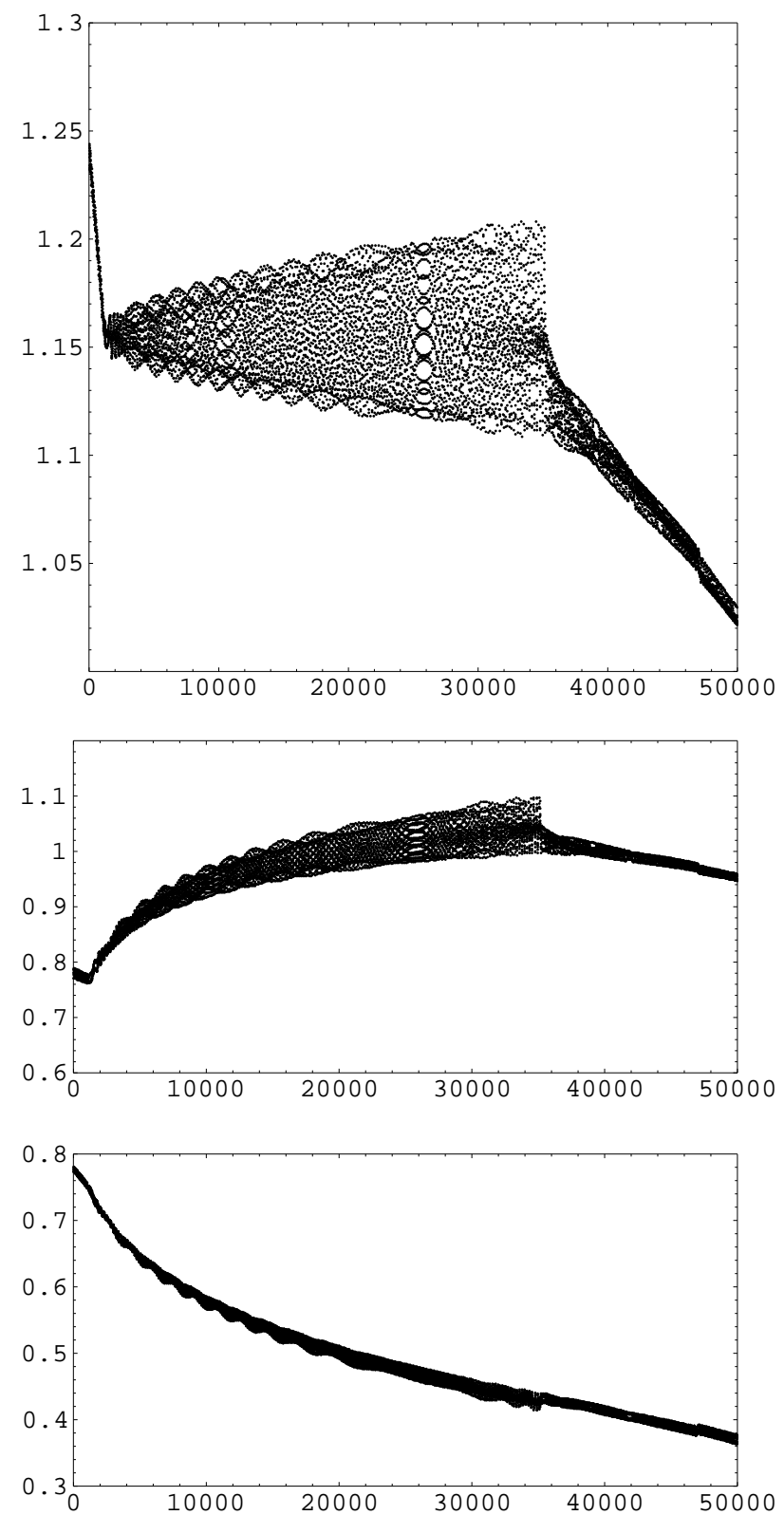

Figure 5. As figure 4 except that the incident radiation has positive helicity. The duration of the resonance is $\approx 34 / \epsilon$ time units; this is consistent with corollary 4.2 . The response of the system is more pronounced in this case, since the sense of the orbital angular momentum is the same as the helicity of the incident radiation.

dissipation; nevertheless, the analysis of the amplitude of oscillations around the resonance is very similar to that carried out in section 6 of [6] due to the fact that $\tau<|\lambda|$.

Figures 4 and 5 also illustrate the complex structure that is usually associated with a higherorder resonance [4]; furthermore, the presence of chaos is strongly indicated in our numerical work. Chaos is especially noteworthy in figure 5; therefore, the qualitative significance of 
our numerical results should be emphasized. We have performed numerical experiments with $\epsilon$ and $\delta$ twice as large as those in figures 4 and 5. In this case, we find in the experiment corresponding to figure 5 that the $(2: 1)$ resonance appears to have significant overlap with the $(3: 1)$ resonance. Let us note that we expect $\epsilon$ to be many orders of magnitude smaller in physical situations of interest; however, we have taken $\epsilon$ to be $10^{-3}$ in figures 4 and 5 for the sake of simplicity.

Do all relativistic binary orbits monotonically decay by gravitational radiation damping? There is evidence for such energy loss from the timing analysis of only two pulsar systems [11]. However, such a system could in principle fall into sustained resonance with an external source; during resonance lock, the orbit would not decay on the average. The orbital decay would resume once the system is released from resonance. It remains to see-as more binary pulsars are discovered and their timing data accumulate - whether sustained resonance in fact occurs in relativistic binary systems.

\section{Acknowledgments}

Carmen Chicone was supported by the NSF grant DMS-9531811 and the University of Missouri Research Board.

\section{References}

[1] Chicone C, Mashhoon B and Retzloff D G 1996 Gravitational ionization: periodic orbits of binary systems perturbed by gravitational radiation Ann. Inst. H. Poincaré, Phys. Théor. 64 87-125

[2] Chicone C, Mashhoon B and Retzloff D G 1996 On the ionization of a Keplerian binary system by periodic gravitational radiation J. Math. Phys. 37 3997-4016

Chicone C, Mashhoon B and Retzloff D G 1997 J. Math. Phys. 38544 (addendum)

[3] Chicone C, Mashhoon B and Retzloff D G 1997 Gravitational ionization: a chaotic net in the Kepler system Class. Quantum Grav. 14 699-723

[4] Chicone C, Mashhoon B and Retzloff D G 1997 Evolutionary dynamics while trapped in resonance: a Keplerian binary system perturbed by gravitational radiation Class. Quantum Grav. 14 1831-50

[5] Chicone C, Mashhoon B and Retzloff D G 1999 Chaos in the Kepler system Class. Quantum Grav. 16 507-27

[6] Chicone C, Mashhoon B and Retzloff D G 1999 Chaos in the Hill system Helv. Phys. Acta 72 123-57

[7] Henrard J 1985 Resonance sweeping in the solar system Stability of the Solar System and its Minor Natural and Artificial Bodies ed V G Szebehely (Dordrecht: Reidel) pp 183-92

Beaugé C, Aarseth S J and Ferraz-Mello S 1994 Resonance capture and the formation of the outer planets Mon. Not. R. Astron. Soc. 270 21-34

Melita M D and Woolfson M M 1996 Planetary commensurabilities driven by accretion and dynamical friction Mon. Not. R. Astron. Soc. $280854-62$

Winter O C and Murray C D 1997 Resonance and chaos. I. First-order interior resonances Astron. Astrophys. 319 290-304

Haghighipour N 1999 Dynamical friction and resonance trapping in planetary systems Mon. Not. R. Astron. Soc. 304 185-94

Haghighipour N 1999 Resonance lock and planetary dynamics PhD Thesis University of Missouri-Columbia

[8] Wardell Z E 1999 Gravitational radiation reaction and the three body problem (in preparation)

[9] Kopeikin S M 1998 Private communication

Kopeikin S M 1997 Millisecond and binary pulsars as nature's frequency standards. I. A generalized statistical model of low-frequency timing noise Mon. Not. R. Astron. Soc. 288 129-37

Kopeikin S M 1999 Millisecond and binary pulsars as nature's frequency standards. II. Effects of low-frequency timing noise on residuals and measured parameters Mon. Not. R. Astron. Soc. 305 563-90

[10] Bombelli L and Calzetta E 1992 Chaos around a black hole Class. Quantum Grav. 9 2573-99

[11] Stairs I H, Arzoumanian Z, Camilo F, Lyne A G, Nice D J, Taylor J H, Thorsett S E and Wolszczan A 1998 Measurement of relativistic orbital decay in the PSR B1534+12 binary system Astrophys. J. 505 352-7

Taylor J H and Weisberg J M 1989 Astrophys. J. 345 434-50 
[12] Kovalevsky J 1967 Introduction to Celestial Mechanics Astrophysics and Space Science Library vol 7 (New York: Springer)

[13] Sternberg S 1969 Celestial Mechanics vol 1-2 (New York: Benjamin)

[14] Fenichel N 1971 Persistence and smoothness of invariant manifolds for flows Indiana Univ. Math. J. 21 193-226

[15] Robinson C 1983 Sustained resonance for a nonlinear system with slowly varying coefficients SIAM J. Math. Anal. 14 847-60 